\title{
Fazit: Prozessmanagement als Gestaltungshebel der digitalen Transformation?
}

Elke Brucker-Kley, Thomas Keller und Denisa Kykalová

9.1 Status Quo «Kundennutzen durch Digitale Transformation»: Fazit aus der Online-Befragung - 128

9.2 Erfolgsmuster: «Kundennutzen durch Digitale

Transformation» - Fazit aus den Fallstudien - 131

9.3 BPM Quo Vadis? - Prozessmanagement als

Gestaltungshebel der Digitalen Transformation - 133

Literatur - 135 
Die Studie kommt zum Schluss, dass das Hype-Thema «digitale Transformation» die Chance bietet, das Prozessmanagement in einem neuen Licht zu betrachten. Der Beitrag, den das Prozessmanagement zur Gestaltung des digitalen Wandels leisten kann, ist unbestritten. Isoliert sind die damit verbundenen Herausforderungen jedoch nicht zu bewältigen. Das Prozessmanagement muss sich aktiv mit den Paradigmen, Methoden und Werkzeugen anderer Managementdisziplinen auseinandersetzen. Gelingt es Synergien mit den Kräften des Innovationsmanagements, Enterprise Architecture Managements, Wissensmanagements und Customer Experience Managements zu nutzen, können Chancen, aber auch Grenzen der Prozessdigitalisierung sehr viel wirksamer ausgelotet werden. Ein derart technologienahes und datenzentrisches Prozessmanagement, das sowohl die Mitarbeitenden- als auch die Kundenperspektive einnimmt, kann eine aktive Rolle bei der zielgenauen Planung und Gestaltung des digitalen Wandels im Front- und Back-End von Unternehmen spielen.

\subsection{Status Quo «Kundennutzen durch Digitale Transformation»: Fazit aus der Online-Befragung}

Welche Rolle spielt das Prozessmanagement als Gestaltungselement der digitalen Transformation? Ziel der quantitativen Online-Befragung war es, den Status quo des Methoden- und Werkzeugeinsatzes innerhalb und an den Schnittstellen der drei Wirkungsfelder «Prozessmanagement - Kundennutzen - Digitalisierung» zu erheben und daraus Erkenntnisse über die Bereitschafft des Prozessmanagements für den digitalen Wandel abzuleiten.

Die digitale Transformation ist nicht nur ein mediales Phänomen, sondern zeigt sich sehr konkret in einer Vielzahl von Massnahmen und Aktivitäten in den Unternehmen. Dabei wenden sie sich keinesfalls nur der «Sonnenseite» der Digitalisierung in Form mobiler Applikationen oder technologischer Gadgets zu, sondern befassen sich intensiv mit Defiziten in der Durchgängigkeit ihrer Prozesse und mit der Modernisierung ihrer Arbeitsplätze. Das fachliche und technische Prozessmanagement ist also gefordert, aber das volle Potenzial für die Maximierung des Kundennutzens ist noch nicht ausgeschöpft. Die Erkenntnisse aus der quantitativen Online-Befragung (• Abb. 9.1) führen zu folgendem Fazit:

- Strategische Ausrichtung des Prozessmanagements - kundenzentrisch und technologienah?

Transparenz ist immer noch die wichtigste Motivation für das Prozessmanagement. Doch Kundenzufriedenheit gewinnt an Relevanz und wird ähnlich hoch priorisiert wie Effizienz, die mehr als $80 \%$ der Unternehmen als Zielsetzung nennen, aber nur noch rund ein Viertel der Unternehmen als höchste Priorität des Prozessmanagements betrachtet. Unternehmen nutzen die durch das Prozessmanagement gewonnene Transparenz zumindest punktuell, um für die Kundenzufriedenheit kritische Prozesse, Standardisierungs- und Automatisierungspotenzial oder Digitalisierungspotenzial für schwach strukturierte Prozesse oder Kundeninteraktionen $\mathrm{zu}$ identifizieren. Systematisch genutzt werden diese Analysen jedoch von weniger als einem Drittel der befragten Unternehmen. Für das Prozessmanagement besteht also durchaus noch Potenzial, stärkere Impulse für die Digitalisierung und kundenorientierte Gestaltung der Prozesse zu setzen.

- Prozessdigitalisierung - durchgängig und flexibel?

Medienbrüche gehören immer noch zum Alltag in Unternehmen. Durchgängigkeit ist auch nicht unbedingt gegeben, wenn Unternehmen digitale Kanäle optimieren oder erweitern. 44 \% der Unternehmen geben an, keine Methoden einzusetzen, um Prozesse vom und zum Kunden, 


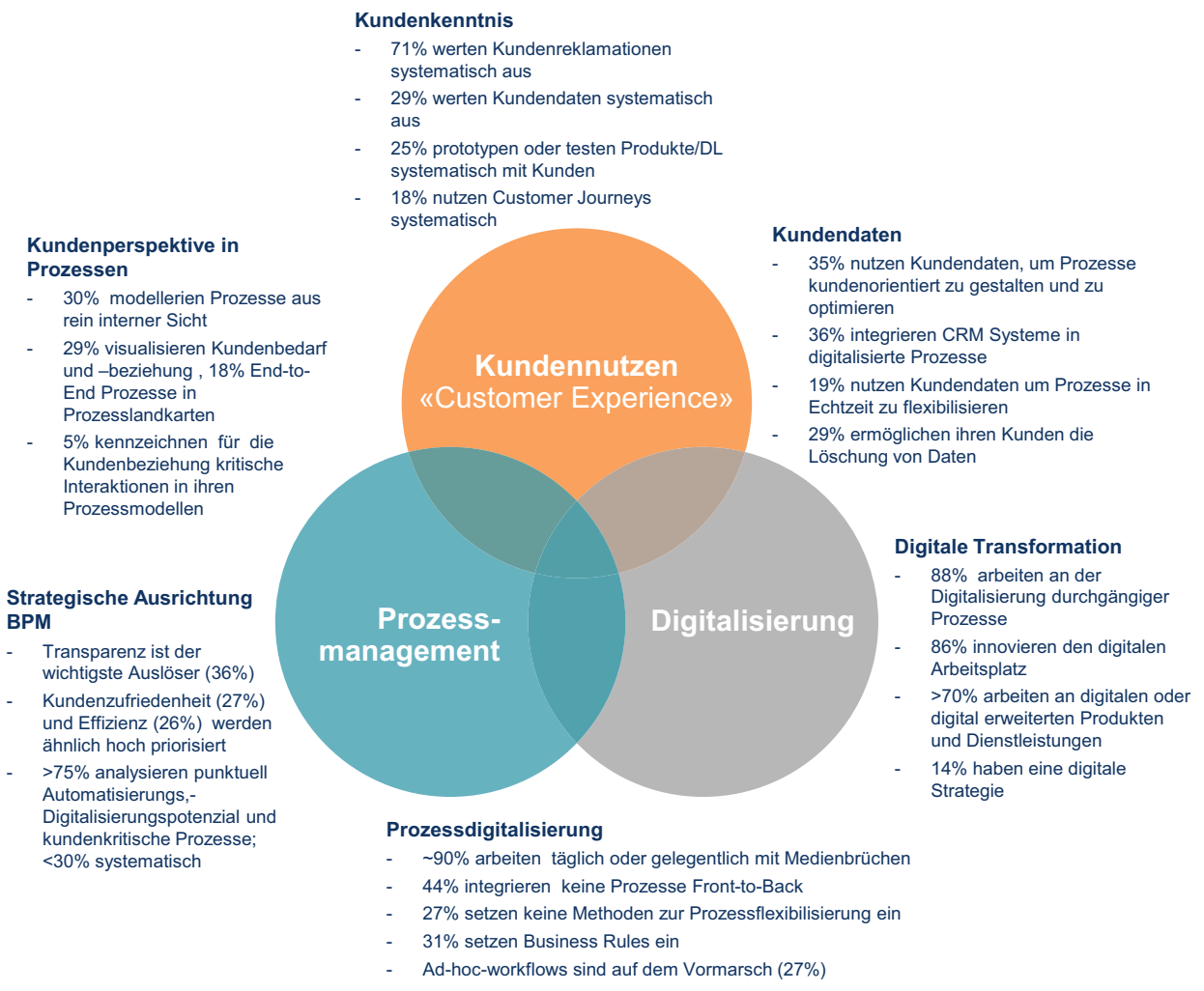

- Abb. 9.1 Zusammenfassung der Ergebnisse der quantitativen Online-Befragung

das heisst Front-to-Back, durchgängig zu realisieren. Dies lässt befürchten, dass viele der aktuell im Rahmen von Digitalisierungsinitiativen realisierten Front-End-Lösungen ohne durchgängige Anbindung an die Back-End-Systeme bleiben und zu Silos werden. Die flexible oder gar individuelle Anpassung von Prozessen an den Kundenkontext ist im Zeitalter der «Customer Experience» noch keinesfalls Alltag. 27 \% der Unternehmen setzen keinerlei Methoden für die Flexibilisierung ihrer Prozesse ein. Wenn sie es tun, dann kommen in Form von Geschäftsregeln etablierte Technologien zum Einsatz, die für komplexe, aber gut formalisierbare Anwendungsfälle greifen. Innovativere, wissensbasierte und selbstlernende Methoden wie beispielsweise Adaptive Case Management, die auch schwach strukturierte und wissensintensive Prozesse digitalisieren könnten, fristen hingegen weiterhin ein Schattendasein. Erfreulich ist der vermehrte Einsatz von Ad-hoc-Workflows im Vergleich zur Studie des Vorjahres, da sie Prozessbeteiligten mehr Freiheit für die Ausgestaltung konkreter Prozessinstanzen in schwach strukturierten Prozessen geben.

\section{- Digitale Transformation - Front-End versus Back-End, strategisch versus opportunistisch?}

Die Hypothese, dass sich Unternehmen aktuell vor allem den medienwirksamen Front-EndThemen der Digitalisierung widmen, hat sich nicht bestätigt. Unternehmen haben offensichtlich erkannt, dass sie sich zunächst den zunehmend dringlichen Durchgängigkeitsdefiziten ihrer Prozesse zuwenden müssen ( 88 \%), bevor sie die «digitalen Tore» zum Kunden aufstos- 
sen können. Auch die Schaffung innovativer digitaler Arbeitsplätze geniesst Vorrang (86\%). Entweder reagieren Unternehmen auf den Druck einer neuen Generation von Mitarbeitenden oder sie sehen Mobilität und moderne Kollaboration als Voraussetzung für eine bessere Interaktion mit Partnern und Kunden. Innovation und Customer Experience treten dabei jedoch nicht in den Hintergrund. Unternehmen arbeiten intensiv an digital erweiterten (81\%) oder neuen digitalen Produkten und Dienstleistungen (72\%) und investieren in neue Technologien an der Schnittstelle zum Kunden (z. B. Mobile, IoT, Social Media) oder experimentieren zumindest mit diesen (77\%). KMUs sind leicht zurückhaltender bei technologie- und kostenintensiven Themen, wie der Optimierung der User Experience, Big Data oder einer serviceorientierten Architektur. Die Frage, ob diese Aktivitäten opportunistisch oder top-down von einer unternehmensweiten digitalen Strategie getrieben sind, lässt sich aktuell nicht abschliessend beantworten. Nur $14 \%$ der Unternehmen haben eine digitale Transformationsstrategie, aber weitere $44 \%$ geben an, an einer solchen zu arbeiten oder zumindest eine solche in Betracht zu ziehen.

\section{- Kundenbedürfnisse - Fragebogen oder Customer Journey?}

Klassische retrospektive und quantitative Methoden, um Kundenbedürfnisse zu erheben, wie die Auswertung von Reklamationen, Kundenzufriedenheitsumfragen oder Kundenzufriedenheitskennzahlen sind immer noch dominant. Methoden, die darauf ausgerichtet sind, Kundenbedürfnisse frühzeitig zu erheben, gewinnen aber durchaus an Relevanz. $25 \%$ «prototypen» oder testen neue Produkte, Dienstleistungen oder Interaktionsformen vor der Markteinführung systematisch. Mehr als die Hälfte der Unternehmen setzen Customer Journeys ein, 18 \% bereits systematisch.

- Kundenperspektive - Was leisten Prozesslandkarten und -modelle?

Obwohl Kundenzufriedenheit ein hoch priorisiertes Ziel des Prozessmanagements ist, werden Prozesslandkarten, die zentralen Kommunikationsinstrumente des strategischen Prozessmanagements, von weniger als einem Drittel der Unternehmen genutzt, um Kundenbedarf und Kundenbeziehungen zu visualisieren. Die konsequente End-to-End-Ausrichtung zeigt sich nur bei $18 \%$ der Prozesslandkarten. $30 \%$ der Unternehmen räumen ein, Prozesse ausschliesslich aus der internen Perspektive zu modellieren. Dass Prozessmodelle damit bedingt einsetzbar sind, um Prozessverantwortliche und Prozessbeteiligte bei der kundenorientierten Optimierung von Prozessen zu unterstützen liegt auch daran, dass kaum Details zu den Kundeninteraktionen hinterlegt werden und auch die für die Kundenbeziehung kritischen Aktivitäten und Interkationen nicht speziell gekennzeichnet werden.

\section{- Kundendaten - Grundlage für die Prozessoptimierung und flexible Prozessausführung?}

Obwohl Unternehmen Kundendaten ausgeprägt sammeln und auswerten, werden sie für die Prozessgestaltung und -ausführung noch nicht umfassend genutzt. Nur etwa ein Drittel der Unternehmen nutzt Kundendaten für die kundenorientierte Prozessoptimierung und -gestaltung. Nur $19 \%$ nutzt Kundendaten, um Prozesse in Echtzeit zu flexibilisieren. Eine Ursache könnte in der noch nicht optimal ausgeprägten Integration der CRM-Systeme in die Prozessausführung liegen. In Sachen Datenherrschaft spüren die Unternehmen grösstenteils noch keinen Druck, die Transparenz und Mitbestimmungsmöglichkeiten ihrer Kunden über das gesetzliche Mindestmass hinaus zu erweitern. 


\subsection{Erfolgsmuster: «Kundennutzen durch Digitale Transformation» - Fazit aus den Fallstudien}

Welchen Beitrag kann das Prozessmanagement - über Standardisierung und Effizienzsteigerung hinaus - für die Gestaltung des technologischen Wandels und die Maximierung des Kundennutzens leisten? Welche Rolle spielt dabei die Digitalisierung von Prozessen und Kundenerlebnissen?

Während die Ergebnisse der Online-Befragung ein rein quantitatives Bild vom Status quo vermitteln, erlaubten die Interviews und der eintägige Praxisworkshop mit fünf Unternehmen einen vertieften Einblick in verschiedene Anwendungsszenarien und Lösungsansätze. Die Interviews mit den Firmenvertretern im Vorfeld des Workshops, die Präsentationen und Diskussionen im Workshop sowie die Analyse und Aufbereitung der Fallstudien im Anschluss (Kap. 4-8) schärften nicht nur die Hypothesen, sondern erlauben Rückschlüsse auf mögliche Erfolgsmuster für den Einsatz des Prozessmanagements als Hebel für den digitalen Wandel. Die identifizierten Erfolgsmuster, eingeordnet in das Rahmenwerk der Studie (Abschn. 1.3) sind nachfolgend zusammengefasst (- Abb. 9.2).

\section{Kundennutzen durch digitale Transformation?}

- Externe und interne Kunden mit neuen digitalen oder digital erweiterten Produkten, Dienstleistungen und Interaktionsmöglichkeiten zu überzeugen, gelingt Unternehmen immer noch, indem sie offensichtliche Defizite beziehungsweise Optimierungspotenziale erkennen und adressieren. Kürzere Reaktionszeiten, im Idealfall Echtzeit-Reaktionen und mehrstufige Prozesse oder Wege, die entfallen (eUmzugZH, AMAG), oder eine verbesserte Service- und Beratungsqualität durch die optimierte Verfügbarkeit von Informationen (Swiss Re), mögen keinen «Wow-Effekt» erzeugen, werden von Kunden in allen untersuchten Fallstudien aber geschätzt und noch nicht als selbstverständlich empfunden. Möglich ist dies immer dann, wenn durch diese Optimierungen für Kunden ein relativer Nutzen im Vergleich zu Erfahrungen in der Vergangenheit oder mit anderen Dienstleistungen oder Anbietern entsteht. Kundennutzen als relative Grösse anzuerkennen, hilft Unternehmen, auch ihre digitalen Transformationsaktivitäten zu relativieren, das heisst, abhängig von Reifegrad einer Branche oder eines Marktes pragmatisch vorzugehen und in einem ersten Schritt naheliegende spürbare Verbesserungen für Kunden zu realisieren.

- Betreten Unternehmen mit der Digitalisierung einer Dienstleistung Neuland (AMAG Leasing, eUmzugZH), braucht es hingegen andere Ansätze. In diesen Fällen führt die frühzeitige Einbeziehung der Kundenperspektive zum Erfolg. Kundenbedürfnisse lediglich anzunehmen und in Anforderungen zu formulieren, genügt in diesen Fällen nicht. Interviews zu Haltungen und Erwartungen, Prototyping und Testen verschiedener Szenarien mit Kunden, bevor ein digital erweitertes oder vollständig digitalisiertes Angebot finalisiert und eingeführt wird, stellt sicher, dass Einstiegspunkte, Prozesse und Oberflächen bedarfsgerecht und intuitiv gestaltet werden.

- Die Reduktion von Komplexität steht im Mittelpunkt von Initiativen, die in erster Linie auf Effizienzsteigerung aus interner Perspektive ausgerichtet sind. In der Wirkung zeigt sich jedoch, dass auch Kunden von Standardisierung und Harmonisierung profitieren, indem Leistungen, einfacher zugänglich, transparenter und verständlicher werden (Vontobel, Swisscom). Einfachheit und Konsistenz erzeugen Kundennutzen und lassen sich als Erfolgsmuster auch auf externe Kunden anwenden. 


\section{Durchgängige Prozesse für Operational \& Service Excellence}

- Positive Kundenerlebnisse entstehen, wenn Prozesse durchgängig sind. Gleichzeitig lieferten Defizite in der Durchgängigkeit der Prozesse in allen vorliegenden Fallstudien überzeugende Business Cases für die digitale Transformation.

- Synchrone Kommunikation mit Kunden in digitalen Kanälen zeichnet überzeugende Online-Angebote aus, die Kunden ohne Verzögerung und in einem Schritt die gewünschten Leistungen bereitstellen (AMAG, eUmzugZH). Dies setzt jedoch ein hohes Mass an Automatisierung, implementierter Geschäftslogik (z. B. durch Geschäftsregeln), hohe Datenqualität und Integration im Back-End voraus (Amag, Swiss Re). Interne Kunden und Supportprozesse sind dabei nicht zu vernachlässigen. Ist es Unternehmen gelungen, diese bereits durchgängig zu digitalisieren, können die Erfahrungen und Infrastrukturen als Grundlage für überzeugende Serviceprozesse auch für externe Kunden dienen (Vontobel).

- Integration ist ein Erfolgsschlüssel in allen vorliegenden Fallstudien. Gelingt es CRMSysteme oder Kundendatenbanken, interne und externe Systeme, Datenquellen und Ablagen auf effiziente Art und Weise zu integrieren, beschleunigt dies die Implementierung durchgängiger Prozesse. Service-orientierte Architekturen (AMAG, eUmzugZH) und plattformbasierte Ansätze über BPM-Lösungen (Swiss Re) stellen effiziente und unternehmensweite Grundlagen für die Prozessintegration zur Verfügung.

\section{Produkt- \& Service-Innovationen befähigt durch ein digitalisiertes Rückgrat}

- Unternehmen, die Prozesse im Back-End bereits durchgängig digitalisiert haben, können Innovationschancen im Front-End rascher ergreifen und sich so Wettbewerbsvorteile sichern (AMAG). Werden solche Potenziale erkannt, können sich Geschäftsbereiche oder -funktionen neu positionieren und ihre Rolle in der Organisation beziehungsweise der Wertschöpfungskette verändern (AMAG, Vontobel).

- Moderne und intuitive digitale Arbeitsplätze sind wichtige Voraussetzungen für Unternehmen, deren Strategien auf die Erschliessung neuer Märkte und Kundensegmente ausgerichtet sind (Swiss Re, Vontobel). Neue, häufig global verteilte Mitarbeitende sind so rasch produktiv und können in global harmonisierten Prozessen und Instrumenten kollaborieren und Kunden bedienen. Unternehmen, die den digitalen Arbeitsplatz auf diese Weise innovieren, finden das optimale Mass an Standardisierung und Flexibilisierung, um Wissensarbeitende von Routinearbeiten zu entlasten und in ihren Entscheidungen zu unterstützen.

- Auch die Standardisierung in Bereitstellungsprozessen kann Produkt- und Service-Innovationen unterstützen, indem sie die Time-to-Market verkürzt (Swisscom). Dabei wenden Unternehmen Ansätze aus der industriellen Fertigung nicht nur auf Prozesse, sondern auch auf Produkte und Dienstleistungen an. Resultate sind Produkt- und Dienstleistungskataloge mit einer grossen Leistungsbreite und -tiefe, aus denen im Baukastenprinzip Leistungen individuell kombiniert und bezogen werden können.

- Plattformen, die Partner vernetzen, um neue Dienstleistungen und Produkte zu erbringen und durchgängige Prozesse über die Unternehmensgrenzen hinaus mit Partnern in der Wertschöpfungskette ermöglichen, können das Geschäftsmodell erweitern oder verändern. Im öffentlichen Sektor lassen sich die Ergebnisse solcher E-Government-Innovationen in Referenzmodellen abbilden und so deren flächendeckende Ausdehnung für die Bevölkerung beschleunigen (eUmzugZH). 


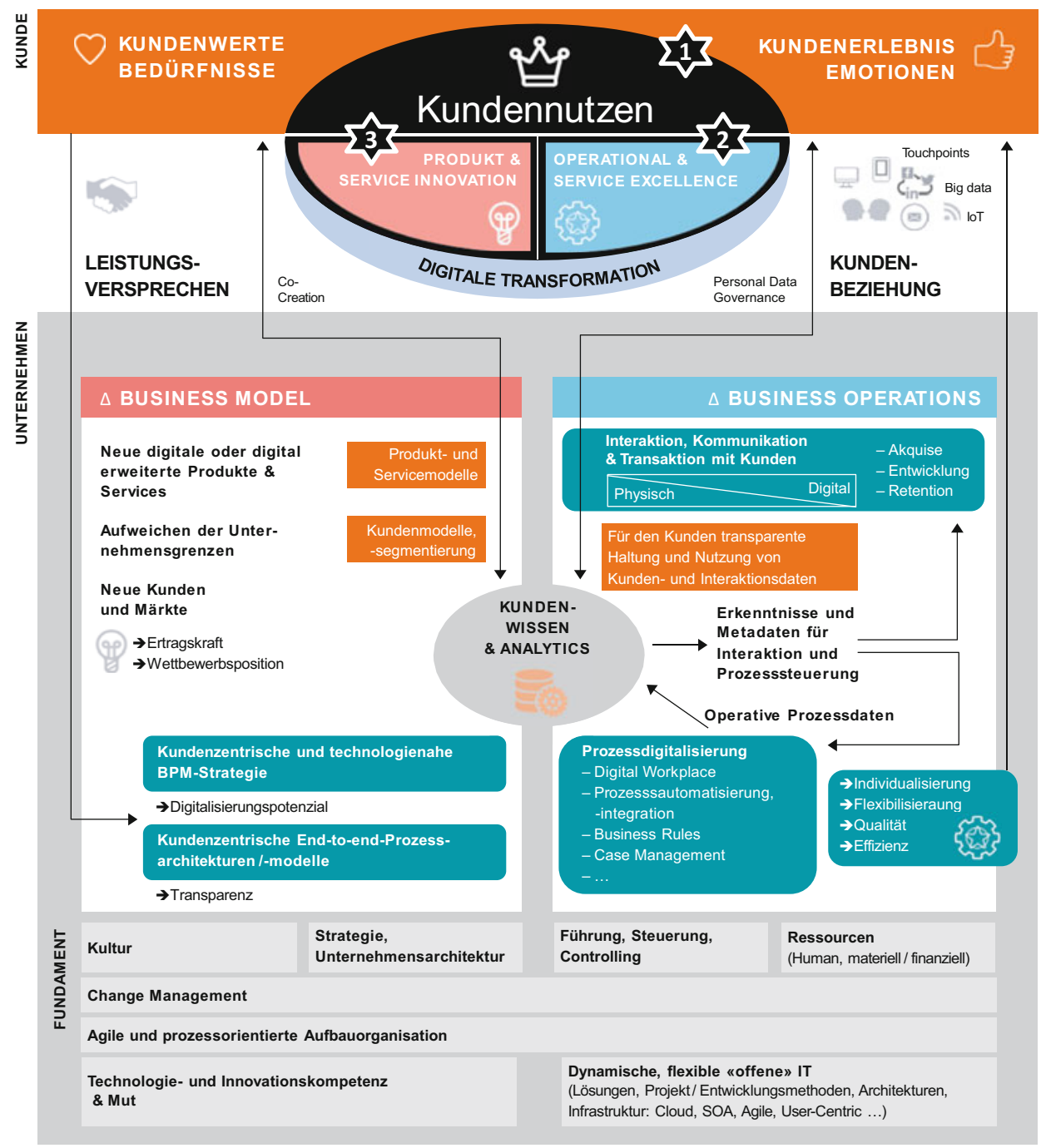

- Abb. 9.2 Kundennutzen durch digitale Transformation? - Rahmenwerk der Studie

\subsection{BPM Quo Vadis? - Prozessmanagement als Gestaltungshebel der Digitalen Transformation}

Bietet das Hype-Thema «digitale Transformation» die Chance, Prozessmanagement in einem ganz neuen Licht zu betrachten? So lautete die Eingangsfrage der BPM-Studie 2016. Der Beitrag, den das Prozessmanagement zur Gestaltung des digitalen Wandels leisten kann, ist unbestritten. Isoliert sind die damit verbundenen Herausforderungen jedoch nicht zu bewältigen. Das Prozessmanagement muss sich aktiv mit den Paradigmen, Methoden und Werkzeugen anderer Managementdisziplinen auseinandersetzen. Gelingt es Synergien mit den Kräften des Innovationsmanagements, Enterprise Architecture Managements, Wissensmanagements und 
Customer Experience Managements zu nutzen, können Chancen, aber auch Grenzen der Prozessdigitalisierung sehr viel wirksamer ausgelotet werden (- Abb. 9.3).

- Technologienah und strategisch - BPM und Geschäftsarchitekturen als Impulsgeber Transparenz und Effizienz sind die etablierten Gradmesser für die Wirksamkeit des Prozessmanagements. Unternehmen, die bereits über ein strategisch ausgerichtetes, unternehmensweites Prozessmanagement sowie Prozesslandkarten verfügen, können diese wertvolle Ausgangsbasis nutzen, um Digitalisierungspotenziale systematisch zu identifizieren. Voraussetzung ist eine enge Verzahnung der Prozessarchitektur und -modelle mit der Geschäfts- und Informationssystemarchitektur. Die konzertierte Modellierung von Prozessen, Geschäftsfähigkeiten, Rollen, Stakeholdern, Zielen bis hin zu Geschäftsobjekten und Business Services im Kontext des Enterprise Architecture Management kann die Identifikation relevanter Business Cases für die Prozessdigitalisierung systematisieren und deren nahtlose Spezifizierung in implementierungsfähige Use Cases beschleunigen. In diesem Modus kann das Prozessmanagement auch Impulse für das Innovationsmanagement in interdisziplinären Teams geben.

- Flexibel und transparent - «Data-Centric BPM»

Bereits in der BPM-Studie 2015 zum Thema «Prozessintelligenz» wurde die schwach ausgeprägte Nutzung operativer Prozessdaten in Unternehmen thematisiert (Brucker-Kley et al. 2015). Die Studie 2016 bestätigt dieses Defizit in Bezug auf die Nutzung von Kunden- und Bewegungsdaten für die Optimierung und Flexibilisierung von Prozessen. Reaktionsfähigkeit in Echtzeit bedeutet hohe Anforderungen and die Daten- und Prozessintegration. Das fachliche und operative Prozessmanagement kann wertvolles Wissen über Kunden nur nutzen, wenn es Kompetenzen mit den Disziplinen Business Intelligence und Data Governance bündelt. Nicht ausser Acht gelassen werden darf dabei der Anspruch der Kunden auf eine selbstbestimmte und transparente Haltung und Nutzung der Daten.

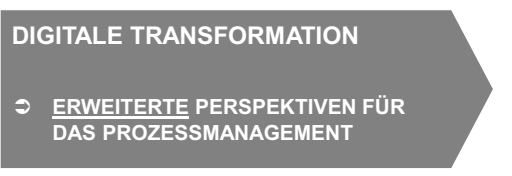

Von Transparenz zu

Digitalisierungsimpulsen

Von aktivitätenzentrisch zu datenzentrisch

Von der rein internen operativen Sicht hin zur

Kundenperspektive

Von Kontrolle, Automatisierung und Standardisierung hin zur Befähigung von Mitarbeitenden

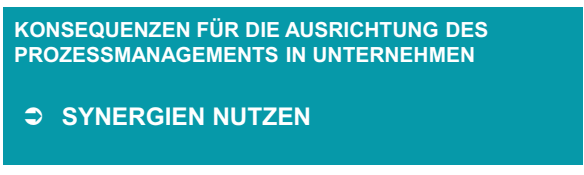

BPM \& Innovationsmanagement BPM \& IT Strategie, Enterprise Architecture

BPM \& Business Intelligence BPM \& SOA / EAI

BPM \& Service Design, Customer Experience Management BPM \& User Experience Design

BPM \& Knowledge Management BPM \& Künstliche Intelligenz

- Abb. 9.3 BPM quo vadis? Entwicklungspotenziale im Kontext des digitalen Wandels 
- Wissenbasiert und intuitiv - «Employee-Centric BPM»

Die Schaffung innovativer und attraktiver «Digital Workplaces» ist ein aktuell intensiv bearbeitetes Aktionsfeld von Unternehmen im Kontext der digitalen Transformation. Einerseits haben Unternehmen also erkannt, dass moderne Arbeitsplätze eine wesentliche Voraussetzung für modernes und kundenorientiertes Arbeiten darstellen. Andererseits fristen selbstlernende Methoden (z. B. Adaptive Case Management), die Wissensarbeitende im Push-Modus mit relevanten Informationen und Handlungsalternativen versorgen, immer noch ein Schattendasein. Will das Prozessmanagement einen Beitrag zur Unterstützung wissensintensiver Prozesse und attraktiver digitaler Arbeitsplätze leisten, muss es den Horizont über die Automatisierung standardisierter Prozesse hinaus in Richtung Knowledge Management und Künstliche Intelligenz erweitern.

\section{- Perspektivenwechsel - «Customer-Centric BPM»}

Unternehmen setzen zunehmend Methoden ein, um Kundenerlebnisse zu verbessern und Kundenbedürfnisse frühzeitig in die Entwicklung neuer Produkte und Dienstleistungen einzubeziehen. Die konsequente Einnahme der Kundenperspektive ist bei der Entwicklung von Personas oder Customer Journeys selbstverständlich. Das Prozessmanagement hingegen entwirft und optimiert Prozesse in der Regel aus rein interner, operativer Perspektive. Herausragende Kundenerlebnisse bedingen jedoch sowohl eine optimale Gestaltung der Customer Touchpoints als auch durchgängige Prozesse. Die Potenziale eines Zusammenwirkens von Prozessmanagement und Marketing sowie Customer Experience Management gilt es daher zu nutzen, unter anderem um besser abzuschätzen, wann und wo Prozessdigitalisierung den Kundennutzen erhöhen oder gar minimieren oder zerstören kann.

\section{Literatur}

Brucker-Kley, E., Kykalová, D., Grünert, D., Keller, T., Schertenleib, R., Schlatter, U., \& Schwer, K. (2015). Business Process Management 2015: Status quo und Best Practices »Prozessintelligenz« - Eine Studie des Instituts für Wirtschaftsinformatik. Winterthur: ZHAW School of Management and Law.

Open Access Dieses Kapitel wird unter der Creative Commons Namensnennung 4.0 International Lizenz (http://creativecommons.org/licenses/by/4.0/deed.de) veröffentlicht, welche die Nutzung, Vervielfältigung, Bearbeitung, Verbreitung und Wiedergabe in jeglichem Medium und Format erlaubt, sofern Sie den/die ursprünglichen Autor(en) und die Quelle ordnungsgemäß nennen, einen Link zur Creative Commons Lizenz beifügen und angeben, ob Änderungen vorgenommen wurden.

Die in diesem Kapitel enthaltenen Bilder und sonstiges Drittmaterial unterliegen ebenfalls der genannten Creative Commons Lizenz, sofern sich aus der Abbildungslegende nichts anderes ergibt. Sofern das betreffende Material nicht unter der genannten Creative Commons Lizenz steht und die betreffende Handlung nicht nach gesetzlichen Vorschriften erlaubt ist, ist für die oben aufgeführten Weiterverwendungen des Materials die Einwilligung des jeweiligen Rechteinhabers einzuholen. 